\title{
Deterministic Writing and Control of the Dark Exciton Spin Using Single Short Optical Pulses
}

\author{
I. Schwartz, ${ }^{1}$ E. R. Schmidgall, ${ }^{1}$ L. Gantz, ${ }^{1}$ D. Cogan, ${ }^{1}$ E. Bordo,${ }^{1}$ Y. Don,,${ }^{1}$ M. Zielinski, ${ }^{2}$ and D. Gershoni ${ }^{1}$ \\ ${ }^{1}$ The Physics Department and the Solid State Institute, \\ Technion-Israel Institute of Technology, 32000 Haifa, Israel \\ ${ }^{2}$ Institute of Physics, Faculty of Physics, Astronomy and Informatics, Nicolaus Copernicus University, \\ ul Grudziadzka 5, PL-87-100 Torun, Poland \\ (Received 10 April 2014; revised manuscript received 3 December 2014; published 30 January 2015)
}

\begin{abstract}
We demonstrate that the quantum dot-confined dark exciton forms a long-lived integer spin solid-state qubit that can be deterministically on-demand initiated in a pure state by one optical pulse. Moreover, we show that this qubit can be fully controlled using short optical pulses, which are several orders of magnitude shorter than the life and coherence times of the qubit. Our demonstrations do not require an externally applied magnetic field, and they establish that the quantum dot-confined dark exciton forms an excellent solid-state matter qubit with some advantages over the half-integer spin qubits, such as the confined electron and hole, separately. Since quantum dots are semiconductor nanostructures that allow integration of electronic and photonic components, the dark exciton may have important implications for implementations of quantum technologies consisting of semiconductor qubits.
\end{abstract}

DOI: 10.1103/PhysRevX.5.011009

\section{INTRODUCTION}

The ability to coherently control and exploit matter quantum systems is essential for realizations of future technologies based on quantum information processing (QIP) [1,2]. Optical approaches, in particular, are preferred since they are state selective, require no contacts, and are ultrafast [3]. Semiconductors play an important role among the matter venues of choice, since they dovetail with contemporary leading technologies, in general, and those of light sources and detectors, in particular. For these reasons, optical control of spins of charge carriers in semiconductor quantum dots (QDs) has been the subject of many recent works [4-10]. While self-assembled QDs still leave unresolved the issues of scalability required for QIP due to the stochastic nature of their size, shape, and composition, these systems do provide a convenient platform for extensive investigations of single- and few-carrier matter qubit systems with extremely efficient coupling to light.

The absorption of a photon in semiconductors promotes an electron from the full valence band to the empty conduction band. This fundamental excitation is particularly efficient because the valence band is formed from molecular $p$-like electronic orbitals, while the conduction band is formed from $s$-like orbitals, and the dipole moment between these orbitals strongly interacts with the electric

Published by the American Physical Society under the terms of the Creative Commons Attribution 3.0 License. Further distribution of this work must maintain attribution to the author(s) and the published article's title, journal citation, and DOI.
Subject Areas: Photonics, Quantum Physics, Quantum Information

field of the light. The photoexcitation, thus, does not alter the promoted electron spin. The excitation leaves an excited electron in the conduction band and a missing electron in the valence band. This many-electron configuration can be described as a two-body state if the missing electron is treated as a hole with opposite quantum numbers (positive rather than negative charge and spin up rather than spin down) [11]. In this description, an absorbed photon results in an electron-hole $(e-h)$ pair with antiparallel spins, or a bright exciton (BE). In InAs/GaAs self-assembled QDs, the quantum size and the lattice mismatch strain result in a considerable energy difference between holes that have their spin direction aligned with their orbital molecular momentum (heavy holes: total angular momentum projection of $\pm 3 / 2$ on the QD growth direction) and holes with a spin direction that is antiparallel to the orbital momentum (light holes: total angular projection of $\pm 1 / 2$ ). The lowest energy BEs are thereby composed of an electron-heavyhole pair with total integer spin projections of \pm 1 on the QD growth axis, reflecting the difference in orbital momentum between the ground (valence) and excited (conduction) electron states. Since the orbital momentum is aligned with the electronic spin, the polarization of light can be straightforwardly used to coherently "write," "read," and manipulate the spin of BEs in self-assembled QDs [12-15].

BEs have many advantages over single half-integer carrier spins, for which deterministic writing and coherent control by a single ultrashort pulse is impossible. Excitons are also advantageous in their electric neutrality, making them less susceptible to decoherence due to electrostatic fluctuations in their vicinity [5-9]. In addition, their 
heavy-hole content partially protects them from decoherence due to nuclear magnetic field fluctuations [8-10,16,17]. Their typically short radiative lifetimes (about $1 \mathrm{nsec}$ ), however, limit their usefulness.

A dark exciton (DE) is an electronic excitation in which the hole spin is parallel to that of the electron. In QDs, the DE has total integer spin of 2 [18], with projections of \pm 2 on the QD growth axis, reflecting the difference in both angular momentum and spin between the conduction and valence band electron states. Since photons barely interact with electronic spin, the DE is almost optically inactive and has a lifetime that is orders of magnitude longer than that of the BE [19].

In this work, we shed new light on the residual optical activity of the DE and directly measure its radiative lifetime. We then circumvent its very weak optical activity and experimentally demonstrate that the QD-confined dark exciton forms a novel semiconductor matter qubit system. We deterministically generate it in one eigenstate using one single optical pulse. We obtain a lower bound of about $100 \mathrm{nsec}$ on its coherence time and use resonant optical pulses to coherently control its spin.

\section{EXPERIMENTAL SYSTEM}

The measurements are carried out on a single straininduced InGaAs QD embedded within a one-wavelength planar microcavity. The microcavity design facilitates efficient collection of the light emitted from single QDs whose optical transitions resonate with the microcavity mode [20]. The sample is grown by molecular beam epitaxy on a [001]-oriented GaAs substrate. One layer of strain-induced InGaAs QDs is deposited in the center of a 285-nm-thick intrinsic GaAs layer. The GaAs layer is placed between two distributed Bragg reflecting mirrors consisting of 25 (bottom) and 10 (top) periods of pairs of AlAs/GaAs quarter-wavelength-thick layers. For the optical measurements, the sample is placed inside a sealed metal tube cooled by a dry helium refrigerator maintaining a temperature of $4.0 \mathrm{~K}$. A $60 \times$ microscope objective with numerical aperture of 0.85 is placed above the sample and used to focus the light beams on the sample surface and to collect the emitted photoluminescence. Continuous wave and/or pulsed laser excitations are used. Acousto-optic modulators are used to produce pulses of few tens of nanoseconds duration. Picosecond pulses are generated by synchronously pumped, cavity-dumped dye lasers. The temporal width of the dye laser pulses is $\sim 10 \mathrm{psec}$ and their spectral width is $\sim 100 \mu \mathrm{eV}$. The polarizations of the pulses are independently adjusted using a polarized beam splitter and two pairs of computer-controlled liquid crystal variable retarders. The polarization of the emitted PL is analyzed by the same liquid crystal variable retarders and polarized beam splitter. The PL is spectrally analyzed by 1-m monochromators and detected by either silicon avalanche photodetectors coupled to a HydraHarp $400^{\mathrm{TM}}$ time-correlated single photon counter or by a cooled charge coupled array detector [21].

\section{RESULTS}

\section{A. Observation of photoluminescence from the dark exciton}

Figure 1(a) presents a PL spectrum of a single QD excited nonresonantly by a $445-\mathrm{nm}(2.78-\mathrm{eV})$ continuous

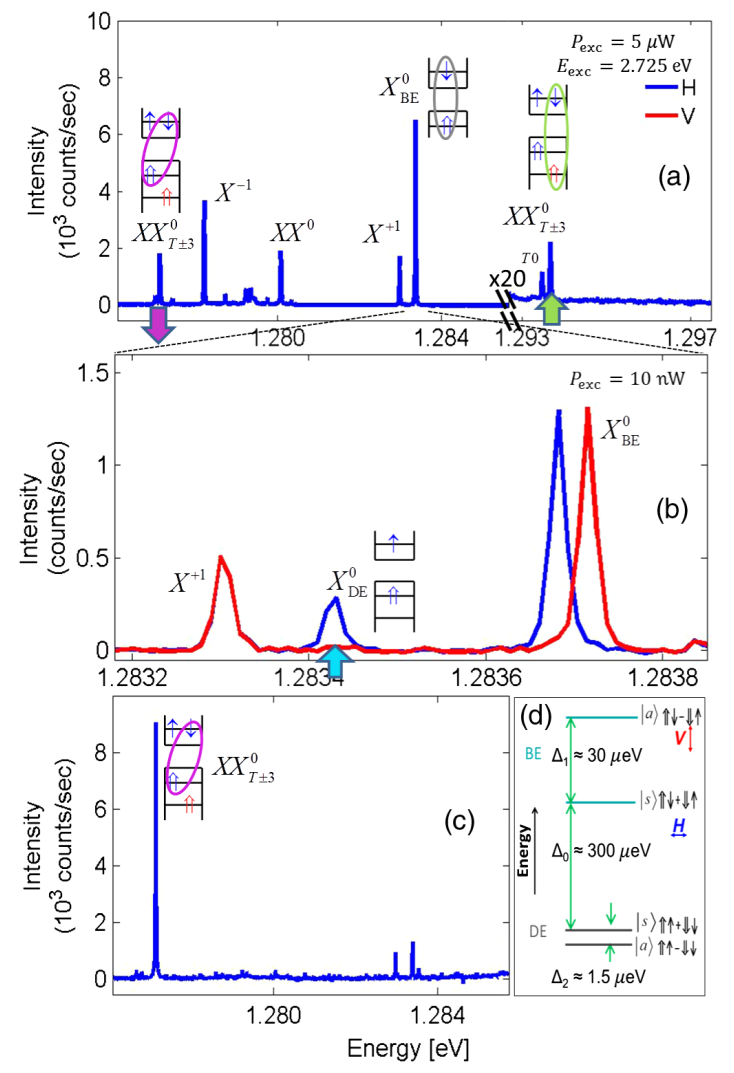

FIG. 1. Photoluminescence spectra of the single QD observation of light emission from the DE. (a) PL spectrum of the QD used in this research excited by high-intensity $(5 \mu \mathrm{W}) \mathrm{cw}$ light. The lines are named by their initial states from which the radiative recombination originates. Above the most relevant lines, carrier configurations are schematically displayed. $\uparrow(\uparrow)$ denotes the electron (heavy-hole) spin state and a short blue (red) arrow denotes $s$ - $(p-)$ shell single carrier level. Only one of the two possible spin configurations is displayed in each case. The recombining $e-h$ pair is indicated by a matching oval. The spectral line used for detecting (exciting) the $X X_{T \pm 3}^{0}$ biexciton line is marked by downward magenta (upward green) arrow. (b) Polarization-sensitive, expanded scale PL spectrum of the QD at low excitation intensity $(10 \mathrm{nW})$. At this excitation level, the DE spectral line is clearly observed about $300 \mu \mathrm{eV}$ below the BE lines. Unlike the BE line, which has two cross rectilinearly polarized components, the DE has one horizontal polarization. (c) PL spectrum from the QD under resonant excitation into the higher-energy optical transition of the $X X_{T \pm 3}^{0}$ biexciton line [upward green arrow in (a)]. (d) Schematic description of the energy fine structure of the QD confined exciton. 
wave laser. Excitation at such a high energy above the band gap photogenerates electron-hole pairs in the bulk semiconductor. The photogeneration rate corresponds to the intensity of the exciting laser. Very small numbers of these carriers, uncorrelated and with randomized spin directions [18], arrive at the QD and populate its lowest energy levels. In $50 \%$ of the cases, $e-h$ pairs with antiparallel spins populate the $\mathrm{QD}$, and $\mathrm{BEs}$ are formed. $\mathrm{A} \mathrm{BE}\left(X_{\mathrm{BE}}^{0}\right)$ recombines radiatively within $\sim 1 \mathrm{nsec}$, giving rise to an observed PL spectral line. Optical recombination of $e-h$ pairs with antiparallel spins from different configurations of charge carriers, such as the charged excitons $X^{+1}$ and $X^{-1}$, results in spectral lines at different energies from different configurations. The identification of these lines has been the subject of substantial research $[13,18,21,22]$.

In this work, we are primarily interested in four of the spectral lines visible in Fig. 1. The initial carrier configurations from which $e-h$ pair recombination gives rise to the observed PL are schematically described above each of these lines. The first emission line $\left(X_{\mathrm{BE}}^{0}\right)$ results from recombination of an electron-hole pair (oval matched in the scheme) in their ground s-shell-like QD levels. The second and third spectral lines initiate from the initial configuration of the spin-blockaded biexciton $\left(X X_{T \pm 3}^{0}\right)$. (This configuration has angular momentum projection of \pm 3 ). It is composed of two electron-heavy-hole pairs, where the electrons form a spin singlet in their ground level and the holes form a spin-parallel triplet with one hole in the QD ground level and one in the first excited level. The line at $\sim 1.277 \mathrm{eV}$ corresponds to recombination of an electron and a ground level hole (oval matched in the scheme), and the much weaker line (about 50 times weaker, due to the reduced overlap between electron and hole wave functions of different symmetries) at $\sim 1.294 \mathrm{eV}$ corresponds to recombination of an electron with the excited hole. In either case, radiative recombination of this biexciton leaves an electron-hole pair with parallel spins, or a dark exciton, in the QD.

As we demonstrate and discuss below, the DE is not totally dark, and it has residual optical activity that results in a very slow radiative decay $[18,19]$. Consequently, at high excitation intensities, the rate of carrier accumulation in the QD exceeds the DE radiative rate by a large margin. Additional carriers destroy the DE, and PL emission from it cannot be observed. In contrast, at very low excitation rates, the carrier accumulation rate is comparable to the $\mathrm{DE}$ radiative rate, and the probability of finding the QD occupied with a DE is very significant. Consequently, if the DE recombines radiatively, its PL emission intensity should become comparable to that of the BE.

Indeed, we demonstrate this in Fig. 1(b). Here, at a nonresonant excitation intensity that is about 3 orders of magnitude weaker than that used in Fig. 1(a), the probability of adding an additional carrier to the QD during the DE lifetime is fairly low, and the PL from the DE spectral line $\left(X_{\mathrm{DE}}^{0}\right)$ is clearly observed. The line marked by an upward blue arrow in Fig. 1(b) is the fourth spectral line of interest.

In Fig. 1(b), we present rectilinear polarization-sensitive PL spectra of the QD at an excitation power that is almost 3 orders of magnitude lower $(10 \mathrm{nW})$ than that used for Fig. 1(a). At $\sim 1.2834 \mathrm{eV}$, approximately $300 \mu \mathrm{eV}$ below the BE lines, a linearly polarized spectral line is clearly seen. The line polarization matches that of the lowest energy component of the $\mathrm{BE}$ ( $H$ along the major axis of the $\mathrm{QD})$, and its energy difference from the BE corresponds well with previous measurements of the DE-BE energy separation in a similar sample [23] and in others [24,25]. Only one linearly polarized transition associated with the $\mathrm{DE}$ recombination is observed. This is in contrast to the $\mathrm{BE}$, which has two almost equally strong cross linearly polarized transitions.

In Fig. 1(c), we present a PL spectrum from the QD under resonant excitation into the higher-energy optical transition of the $X X_{T \pm 3}^{0}$ biexciton line [upward green arrow in Fig. 1(a)] in the presence of very low intensity $(\sim 5 \mathrm{nW})$ nonresonant blue laser excitation. Under these conditions, the $\mathrm{DE}$ is resonantly excited into the $X X_{T \pm 3}^{0}$ biexciton state and the latter most efficiently recombines radiatively, as clearly demonstrated by the dominating emission line due to the lower-energy optical transition of the biexciton [downward magenta arrow in Fig. 1(a)]. The absence or weakness of all other spectral lines indicates that, under these conditions of resonant excitation, the QD remains neutral and it is mainly occupied with DE and its biexciton $X X_{T \pm 3}^{0}$.

Figure 2 presents a measurement of the emission intensities of the $\mathrm{DE}, \mathrm{BE}$, and the two singly charged excitons $X^{+1}$ and $X^{-1}$ as a function of the power of the offresonant excitation. One clearly sees that the DE emission rate saturates at an excitation power that is 3 orders of magnitude lower than the power needed to saturate the emission from the bright excitons (marked by vertical arrows in Fig. 2). Moreover, we note that the maximum emission intensity from the DE is about 3 orders of magnitude weaker than that from the BE (horizontal arrows in Fig. 2). This unambiguously indicates that the DE lifetime is radiative (like that of the $\mathrm{BE}$ ) and that it is 3 orders of magnitude longer $(\sim 1 \mu \mathrm{sec})$ than that of the BE. We verify this straightforward conclusion in two additional independent ways: (1) by measuring the oscillator strength for absorption into the DE line and (2) by directly measuring the DE lifetime after its deterministic initialization.

\section{B. Optical activity of the dark exciton}

The residual optical activity of the DE in rotationally symmetric QDs is attributed to light-hole-heavy-hole mixing. This mixing results in a small, nonvanishing dipole matrix element, polarized along the QD symmetry axis, for 


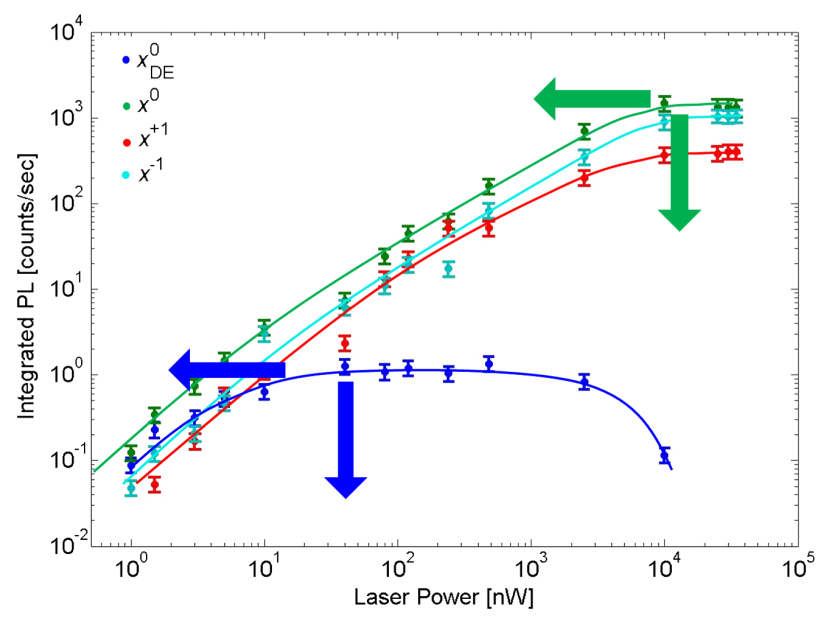

FIG. 2. Emission intensity from various exciton lines as a function of the laser excitation power. Note that the maximum emission of the DE (blue arrow) is $\sim 3$ orders of magnitude weaker than that of the BE (green arrow) and that the power at which the DE maximum occurs is $\sim 3$ orders of magnitude weaker than that of the BE. These measurements demonstrate that the DE decays radiatively with oscillator strength, which is 3 orders of magnitude weaker than that of the BE.

only one of the DE eignestates [26,27]. Realistic atomistic calculations for symmetric InGaAs/GaAs QDs estimate that this DE dipole moment is 5-6 orders of magnitude weaker than that of the $\mathrm{BE}[28-30]$.

In contrast, in Fig. 1(b) we experimentally show a much stronger (only 3 orders of magnitude weaker than the BE) in-plane polarized optical activity of one of the $\mathrm{DE}$ eigenstates. The QD that we report on here (and a few similar ones we measured) is not that symmetric. In reality, self-assembled semiconductor QDs are never ideally symmetric. Their deviation from the combined structural and lattice $C_{2 v}$ symmetry (twofold rotations around the symmetry axis and two reflections about two perpendicular mirror planes that include the symmetry axis) can be due to anisotropic composition and/or strain distribution [24] or to the formation of facets on the interface between the QD and the host material [31]. The influence of this symmetry reduction on the confined $\mathrm{DE}$ and $\mathrm{BE}$ has been quantitatively studied using atomistic model calculations [31]. In short, in a QD with reduced symmetry, BE/DE mixing is anticipated [24]. Only a relatively small such mixing is required for the DE to acquire a small in-plane polarized dipole moment like that of the BEs, while the BE optical activity is hardly affected by the mixing. As the atomistic simulations show [31], the DE/BE mixing has two terms, one due to hole spin flip and one due to electron spin flip. The contributions of these two terms to the optical activity of one of the DE eigenstates is constructive, while to the other eigenstate, it is destructive. If the two mixing terms are comparable, only one DE eigenstate will be optically active. Moreover, if one recalls that by definition the spin of a hole is opposite in direction to that of a missing electron, it follows that the symmetric BE couples to the antisymmetric DE and vice versa.

In order to be consistent with Ref. [32], we define the lowest energy state of the $\mathrm{BE}$ and the direction of its emission as being horizontally linearly polarized $(H)$. From the definition of the $H$ polarization, and the association of the $|+1\rangle,|-1\rangle$ BE states with right- (left-)hand circularly polarized light [12-14], it follows that this choice imposes that the lowest $\mathrm{BE}$ eigenstate is symmetric in the $|+1\rangle$, $|-1\rangle$ basis. Therefore, if one is consistent and uses the basis $|+2\rangle,|-2\rangle$ for describing the DE eigenstates, the optically active DE, which is coupled to the symmetric BE, is antisymmetric in the $|+2\rangle,|-2\rangle$ basis. This being said, we note here that these symmetry properties are basis dependent. We use them in the following for clarity only. Full characterization of an eigenstate is given only by associating it with a measurable property like its energy. We show below, by measuring the direction of the $\mathrm{DE}$ precession, that the optically active DE eigenstate is the lower-energy one. This also agrees with the atomistic model calculations of Ref. [31].

Though only one DE eigenstate is optically active, both of its eigenstates are equally connected through dipoleallowed ("bright") optical transitions to the $X X_{T \pm 3}^{0}$ biexciton states, as described in Fig. 3. Thus, the DE as a physical two-level system (qubit) can be optically accessed and coherently controlled using these optical transitions as we discuss and demonstrate below.

\section{Dark exciton- $X X_{T \pm 3}^{\mathbf{0}}$ biexciton optical transitions}

Figure 3(a) schematically describes the states involved in the experiments and the transitions between these states. The states are represented by horizontal lines in increasing energy order. The carrier configurations, the spin wave function, and the total spin of each state are depicted to the side of each state. Optical transitions between these states are represented by straight arrows whose colors correspond to those used in Figs. 1(a), 3(b)-3(f). The width of the arrows represents the oscillator strength of the transition: the thicker the arrow, the stronger the transition that it represents. Curly gray downward arrows represent nonradiative relaxation of the excited hole to its ground state, and the curved horizontal gray arrows represent coherent Larmor precession of the DE qubit and that of the $X X_{T \pm 3}^{0}$ biexciton. The rates for each transition as directly measured or independently deduced from the experiments are summarized in Table I.

The various steps in our experiments are schematically described in Figs 3(b)-3(f). In the first step [Fig. 3(b)], a resonantly tuned horizontally linearly $(H)$ polarized laser pulse photogenerates the DE in its lower-energy, optically active, antisymmetric eigenstate $|a\rangle$. The pulse is tuned into the vacuum-DE weak optical transition at $1.2834 \mathrm{eV}$ [marked by a blue arrow in Figs. 1(c) and 3 (a)]. As we 

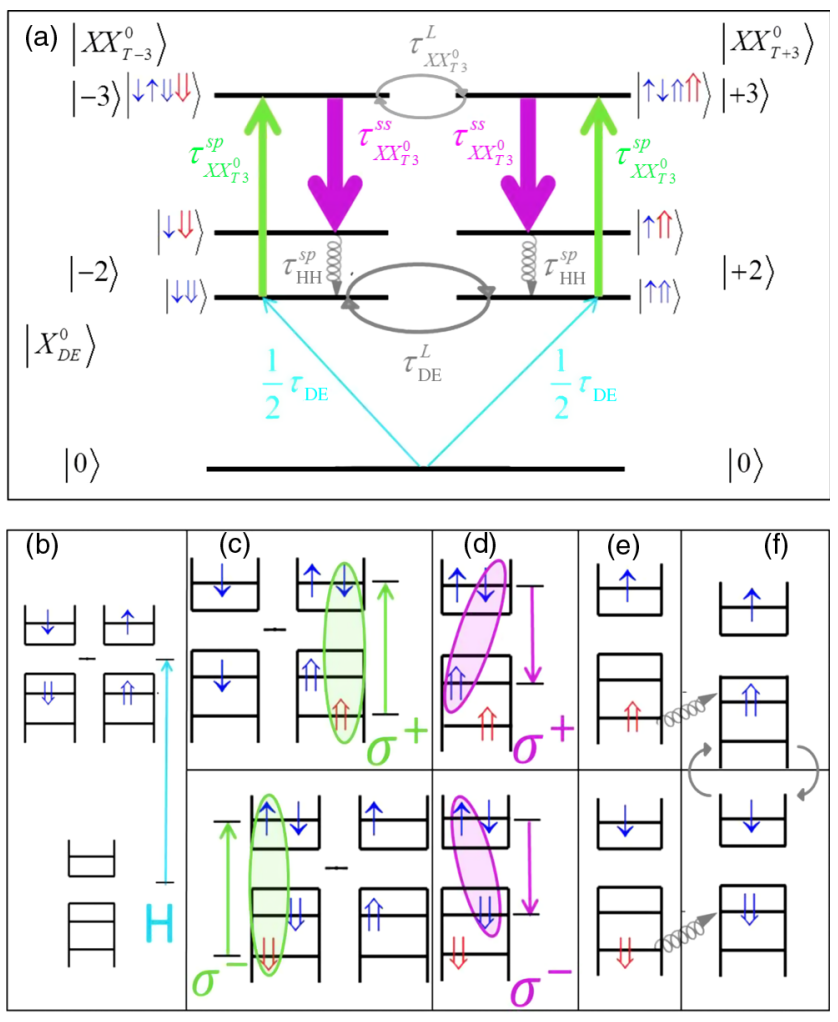

FIG. 3. Schematic description of the experimental steps. (a) The energy levels and optical transitions of the DE-biexciton system are shown schematically. The colors of the arrows correspond to those used in Fig. 1. The width of the arrow represents the strength of the optical transition, with thicker arrows corresponding to stronger dipole moments. Nonradiative transitions are indicated by gray arrows. Curly gray downward arrows indicate nonradiative relaxations, and the curved gray arrows represent coherent precession. The various energies and rates are listed in Table I. (b) An $H$-polarized resonantly tuned laser pulse excites the DE in its antisymmetric eigenstate. (c) A left-hand (bottom panel ) or right-hand (top panel) circularly polarized laser pulse, resonantly tuned to the higher-energy optical transition of the $X X_{T \pm 3}^{0}$ biexciton (green upright arrow in Fig. 1) is used to probe the state of the DE. (d) Detecting a left (bottom panel) or a right (top panel) circularly polarized photon from the lower-energy optical transition of the $X X_{T-3}^{0}\left(X X_{T+3}^{0}\right.$, top) biexciton heralds the presence of a DE in a $|-2\rangle$ (lower panel) or a $|+2\rangle$ (upper panel) coherent state. (e),(f) The heavy hole relaxes to the ground state via a fast spin-preserving relaxation, leaving a ground-state $\mathrm{DE}$ in the QD.

show in the next section, despite the weakness of this optical transition, the intensity and the duration of the pump pulse can be tuned such that a $\pi$ pulse is obtained. In this way, deterministic generation of the DE in a well-defined eigenstate $[|a\rangle=1 / \sqrt{2}(|+2\rangle-|-2\rangle)$, Fig. 3(b) $]$ is achieved.

Probing the $\mathrm{DE}$ presence in the QD is done by absorbtion of a second laser pulse that is resonantly tuned to the $\mathrm{DE}-X X_{T \pm 3}^{0}$ optical transition at $1.294 \mathrm{eV}$ [green arrows in
Figs. 1(a), 3(a), 3(c)]. The efficiency of the absorption is measured by the detection of light emission at $1.278 \mathrm{eV}$ due to the biexciton recombination [downward magenta arrows in Figs. 1(a), 3(a), 3(d)].

As shown in Fig. 3(c), if a DE is present in the QD, a right- (left-)hand circularly polarized $\sigma^{+}\left(\sigma^{-}\right)$probe transfers the $|+2\rangle(|-2\rangle)$ component of the DE population to the $X X_{T+3}^{0}$ or $|+3\rangle\left(X X_{T-3}^{0}\right.$ or $\left.|-3\rangle\right)$ biexciton [Fig. 3(c)]. Note that Pauli exclusion prevents absorption of $\sigma^{-}\left(\sigma^{+}\right)$ photons at this resonance energy when the DE is in the $|+2\rangle(|-2\rangle)$ spin state. Absorption of a given polarization thus directly depends on the DE state. The radiative decay of the $X X_{T+3}^{0}\left(X X_{T-3}^{0}\right)$ biexciton then results in strong $\sigma^{+}$ $\left(\sigma^{-}\right)$emission from the lower-energy transition of this biexciton, which is marked by a downward magenta arrow in Fig. 1(a) [Fig. 3(d)]. The two biexcitonic states, $|+3\rangle$ and $|-3\rangle$, are nearly degenerate in energy, such that there is no appreciable precession between them. We obtain a lower bound of $5 \mathrm{nsec}$ on the Larmor precession time of these two states (Table I). Thus, there is almost a one-to-one correspondence between the DE spin state, the polarization of the absorbed photon, and the polarization of the photon emitted from this biexciton (i.e., a $|+2\rangle$ DE absorbs a $\sigma^{+}$-polarized photon and the corresponding biexcitonic emission is also $\sigma^{+}$polarized, albeit at a different energy). The emission intensity of $\sigma^{+}\left(\sigma^{-}\right)$circularly polarized photons from the $X X_{T \pm 3}^{0}$ biexciton is thus directly proportional to the $\mathrm{DE}|+2\rangle(|-2\rangle)$ state population, and we use this emission for probing both the overall DE population and the specific DE spin state.

While the ground-state electrons in the $X X_{T \pm 3}^{0}$ biexciton could recombine optically with either of the two heavy holes, the biexciton recombination is about 50 times more probable in the lower-energy optical transition [Fig. 1(a)] due to the better overlap of the ground-level wave functions. This recombination leaves a $\mathrm{DE}$ in the QD formed by an electron-excited-hole pair [Fig. 3(e)]. The hole returns to its ground level within $\sim 20$ psec via

TABLE I. Measured times (inverse rates) used in Fig. 3(a). The type of measurement used to derive each quantity is listed in the rightmost column where PL stands for line's intensity ratio, TRS for pump-probe time-resolved spectroscopy, $g^{(2)}$ for intensity correlation measurements, and RECPM for resonantly excited circular-polarization memory measurements.

\begin{tabular}{|c|c|c|c|}
\hline Quantity & Symbol & Time (nsec) & Method \\
\hline BE lifetime & $\tau_{\mathrm{BE}}$ & 0.47 & PL, TRS \\
\hline DE lifetime & $\tau_{\mathrm{DE}}$ & $1.1 \times 10^{3}$ & PL, TRS \\
\hline DE Larmor period & $\tau_{\mathrm{DE}}^{L}$ & 3.1 & $g^{(2)}[18]$ \\
\hline$X X_{T 3}^{0} s p$ radiative time & $\tau_{X X_{T 3}^{0}}^{s p}$ & 6 & PL \\
\hline$X X_{T 3}^{0}$ ss radiative time & $\begin{array}{l}X X_{T 3}^{S S} \\
\tau_{X Y^{0}}\end{array}$ & 0.30 & TRS \\
\hline$X X_{T 3}^{0}$ Larmor period & $\tau_{X X_{T 3}^{A_{T 3}}}^{\Lambda_{T}}$ & $>5$ & RECPM \\
\hline Heavy-hole relaxation time & $\tau_{\mathrm{HH}}^{s p}$ & 0.02 & PL [33], TRS \\
\hline
\end{tabular}


a spin-preserving phonon-mediated relaxation [Fig. 3(f)] $[22,33]$. Consequently, detection of a $\sigma^{+}\left(\sigma^{-}\right)$photon from the biexciton recombination heralds the generation of a $\mathrm{DE}$ in a well-defined coherent superposition of its two eigenstates $|s\rangle$ and $|a\rangle$. The coherent DE then precesses in time [18] on the DE Bloch sphere, as depicted in Fig. 6(a). The precession period of 3.09 nsec corresponds to the energy splitting $(1.4 \mu \mathrm{eV})$ between the DE eigenstates, which has been previously measured [18]. The DE can then be reexcited, using a circularly polarized resonant optical pulse, thereby probing the temporal evolution of its spin state.

\section{Deterministic initialization of the dark exciton and measurement of its lifetime}

We now demonstrate deterministic optical initialization of the DE.

Figure 4(a) displays the PL emission intensity from the $X X_{T \pm 3}^{0}$ biexciton as a function of the power and energy (inset) of the pump laser (represented by a blue arrow in Figs. 1 and 6) in the presence of the probe laser (represented by a green arrow in Figs. 1 and 6). The inset is recorded with continuous wave lasers for two rectilinear polarizations of the pump beam. It demonstrates that the energy and polarization in which the pump laser most efficiently generates the DE is exactly the same as that of the DE observed in photoluminescence spectroscopy $\left[X_{\mathrm{DE}}^{0}\right.$ in Fig. 1(b)]. The linewidth of the DE resonance $(\sim 5 \mu \mathrm{eV})$ reflects the radiative width of the transition to the biexciton, broadened by spectral diffusion caused by the presence of the two laser beams.

In the main panel of Fig. 4(a), pulsed pump-probe measurements at a repetition rate of $1 \mathrm{MHz}$ are used. Rabi oscillations are clearly observed. The pump pulse intensities corresponding to $\pi$ and $2 \pi$ pulses are marked by vertical black arrows. Figure 4(a), therefore, demonstrates that a proper combination of pulse energy, polarization, duration, and power deterministically generates the DE. The deterministic generation of the DE is verified in an additional way. The rate by which single photons due to recombinations of $X X_{T+3}^{0}$ biexciton are detected is in agreement with our estimations based on the known excitation repetition rate $(1 \mathrm{MHz})$ and the overall light harvesting efficiency of the experimental setup (about 1 out of 700 photons).

A comparison between the intensity and temporal pulse width needed to obtain a $\pi$ pulse to the DE resonance with that needed for a $\pi$ pulse to the BE directly yields the ratio between the oscillator strengths (radiative lifetimes) of both excitons. In this way, we independently verify that the DE oscillator strength is more than 3 orders of magnitude weaker than that of the BE.

In Fig. 4(b), we present a direct measurement of the DE lifetime following deterministic initialization of the DE in its $|a\rangle$ spin state. Here, the $X X_{T \pm 3}^{0}$ biexciton emission is measured for various delay times between the DE pump $\pi$ pulse and the probe pulse to the $X X_{T \pm 3}^{0}$ biexciton. The inset describes the experimental sequence of pulses. As stated previously, the intensity of the emitted light from the

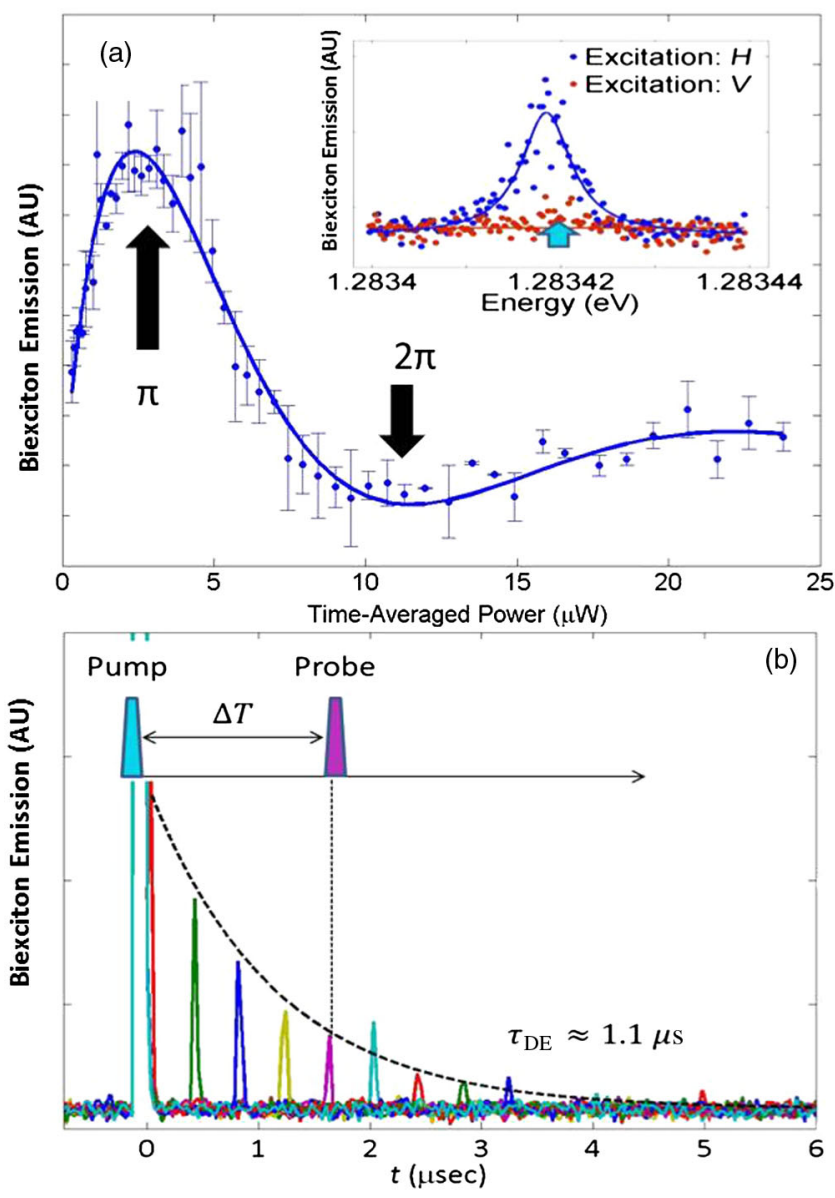

FIG. 4. Deterministic generation of the DE. (a) Lower-energy $X X_{T \pm 3}^{0}$ biexciton emission intensity [Fig. 3(d)] as a function of the average resonant excitation power into the DE absorption resonance [Fig. 3(b)] with a pulse width of $60 \mathrm{nsec}$, in the presence of a probe laser tuned to the higher-energy $X X_{T \pm 3}^{0}$ transition resonance [Fig. 3(c)]. The powers at which $\pi$ and $2 \pi$ pulses are obtained are marked by vertical black arrows. The solid line represents a model fit of the Rabi oscillations. In the inset, the $X X_{T \pm 3}^{0}$ biexciton emission intensity as a function of the energy of the $H(V)$ linearly polarized excitation into the DE optical transition is depicted by the blue (red) dots. The energy used for resonant, deterministic excitation of the DE is indicated by a blue arrow. (b) Lifetime of the DE as measured by a time-resolved double resonant pump-probe experiment. The $X X_{T \pm 3}^{0}$ biexciton emission is measured as a function of the time difference between the DE excitation $\pi$ pulse and the $X X_{T+3}^{0}$ probe pulse. The inset describes the experiment sequence of pulses, where the DE is excited at time $t=0$ (blue pulse) and its population is probed via absorption of a time-delayed probe $\pi$ pulse (magenta). The intensity of the resulting emitted light from the biexciton is a measure of the magnitude of the decaying DE population. The dashed black line is a fitted exponential decay model with characteristic DE lifetime of $1.1 \mu \mathrm{sec}$. 
$X X_{T \pm 3}^{0}$ biexciton probe is a measure of the magnitude of the DE population. The dashed black line is a fitted exponential decay model with a characteristic lifetime of $1.1 \pm 0.1 \mu \mathrm{sec}$. The fact that the measured lifetime of the DE agrees with its measured oscillator strength establishes that the DE decays radiatively from its singly $H$-polarized mode.

\section{E. Coherence time of the dark exciton}

The coherence time of a qubit is an important measure of its decoupling from the environment. Long coherence times are essential for potential applications in QIP. Here, we present a first experimental determination of a lower bound for the coherence time of the DE.

When the DE spin state is a coherent superposition of its two eigenstates $|s\rangle$ and $|a\rangle$, the phase between the two eigenstates varies periodically with a period time (3.09 nsec) [18] given by the Planck constant divided by the energy difference between the eigenstates $(1.4 \mu \mathrm{eV})$. This periodic behavior is commonly described as a precession along the equator of the qubit's Bloch sphere [14] [Fig. 6(a)]. The qubit coherence time can be loosely defined as the characteristic decay time for this precession.

As shown in Fig. 3(c), a $\sigma^{+}\left(\sigma^{-}\right)$probe photon transfers the $|+2\rangle(|-2\rangle)$ component of the DE population to the $X X_{T+3}^{0}\left(X X_{T-3}^{0}\right)$ biexciton. Absorption of a photon of given polarization thus directly depends on the DE spin state. The $X X_{T+3}^{0} \quad\left(X X_{T-3}^{0}\right)$ biexciton radiatively decays within 300 psec, resulting in emission of a $\sigma^{+}\left(\sigma^{-}\right)$polarized photon, which reveals the state of the DE when the probe photon is absorbed. Thus, circular-polarization-sensitive intensity autocorrelation measurements of the the $X X_{T \pm 3}^{0}$ spectral line in the presence of the probe laser provide a direct measurement of the temporal evolution of the DE spin state. The first detected $\sigma^{+}$-polarized photon heralds the DE presence in the $|+2\rangle$ coherent state, while the second detected $\sigma^{+}\left(\sigma^{-}\right)$polarized photon projects the DE on the $|+2\rangle(|-2\rangle)$ state at the time when the probe photon is absorbed.

In Fig. 5(a), black (red) solid lines present intensity autocorrelation measurements of the $X X_{T \pm 3}^{0}$ spectral line under resonant $\mathrm{cw}$ excitation for cocircularly (crosscircularly) polarized photon pairs. By subtracting the cross-circular measurement from the cocircular measurement and normalizing by their sum, the temporal evolution of the degree of circular polarization of the second photon is obtained [Fig. 5(b)]. The observed periodic oscillations in the degree of circular polarization result from the coherent precession of the DE spin state. We note that the maximal degree of polarization obtained is approximately 0.5 . This is expected, considering the limited temporal resolution of our detectors [18]. In addition, we note that the oscillations decay with a characteristic decay time of about $25 \mathrm{nsec}$. This polarization decay is a lower bound on the DE coherence time, since no polarization oscillations can be observed beyond the DE coherence time. Under cw probe excitation, the polarization oscillations also decay for another reason. Under these conditions, the probability that the detected second photon results from the absorption of only one probe

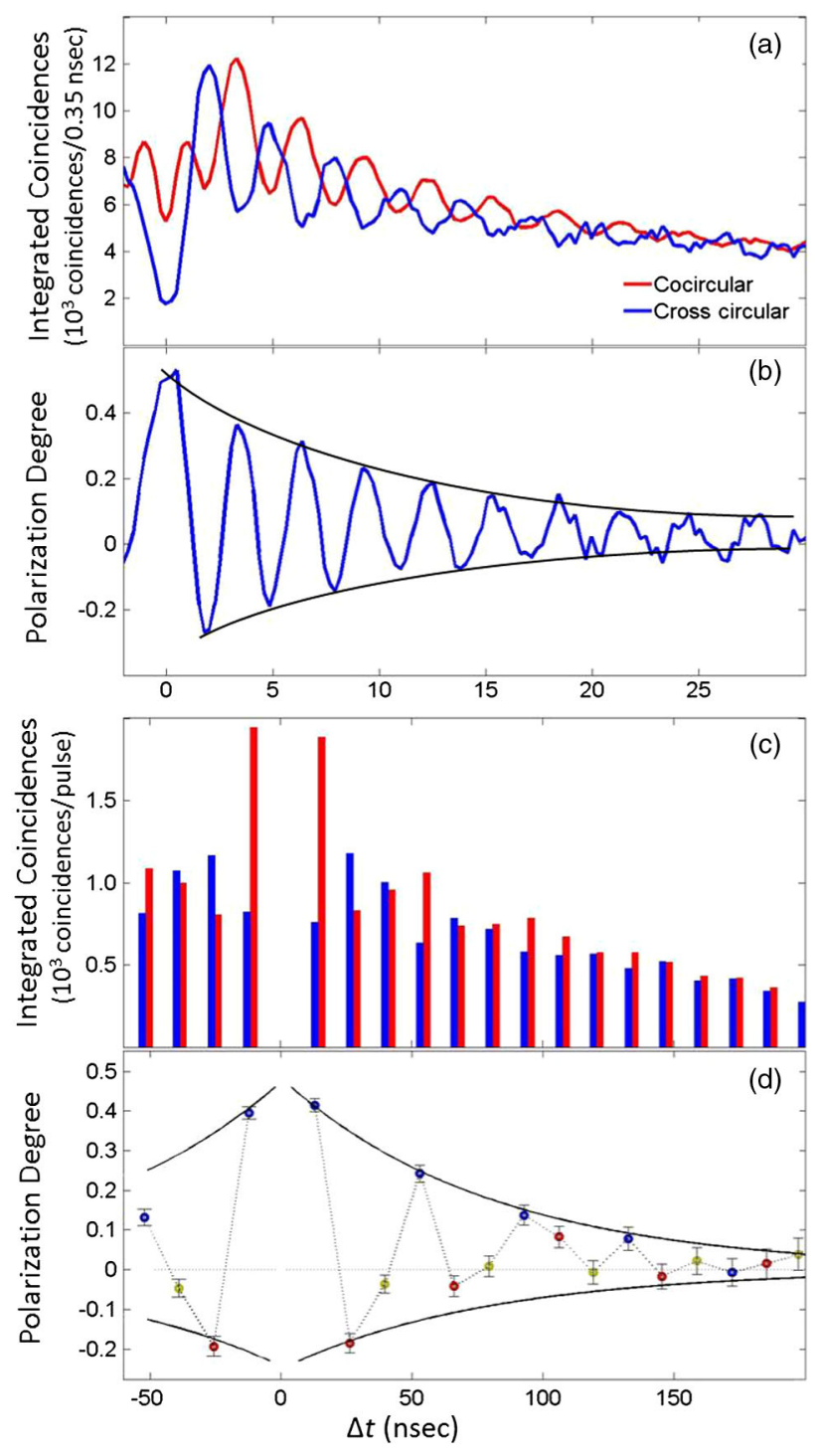

FIG. 5. (a) Polarization-sensitive intensity autocorrelation measurements of the $X X_{T \pm 3}^{0}$ biexciton (vertical magenta arrow in Fig. 1) under resonant $\mathrm{cw}$ excitation (vertical green arrow in Fig. 1). The red (blue) lines display the cocircular (cross-circular) coincidence measurements. (b) The degree of circular polarization of the second detected photon as a function of its detection time as deduced from the measurements in (a). The decay of the polarization oscillations is overlaid by an exponential decay curve (solid black line) with characteristic time of $\sim 25$ nsec. (c) Similar to (a) but under pulsed excitation at a repetition rate of $76 \mathrm{MHz}$. The red (blue) bars display the integrated number of coincidences emitted after each laser pulse for cocircularly (cross-circularly) polarized photons. (d) Like (b) but for the pulsed excitation presented in (c). From the temporal decay of the amplitude of the oscillations (solid black lines), a lower bound of $100 \pm 20 \mathrm{nsec}$ for the DE coherence time is deduced. 
photon, immediately after the photon that heralds the DE, decreases quickly with time. The absorption of each photon followed by spontaneous biexciton recombination, which is not detected, reduces the measured polarization oscillations. Indeed, we observe that the $\mathrm{cw}$ polarization decay time strongly depends on the probe light intensity (not shown).

In Fig. 5(c), we present a similar measurement using pulsed probe light at a repetition rate of approximately $76 \mathrm{MHz}$. Red (blue) bars display the integrated number of cocircularly (cross-circularly) polarized coincidences after each laser pulse. The measured degree of circular polarization of the second photon as a function of the pulse time is given in Fig. 5(d). Since the DE period is about $3 \mathrm{nsec}$ while the laser period is about $13 \mathrm{nsec}$, the periodicity of the measured polarization is expected to be about $3 \times 13 \mathrm{nsec}$, or about three laser pulses. This is exactly what we observe in Fig. 5(d). The maxima (minima) of the oscillations are represented with blue (red) markers and occur every three pulses. The polarization degree decays with a characteristic time of $100 \pm 20 \mathrm{nsec}$. This measurement, therefore, sets a lower bound on the coherence time $T_{2}^{*}$ of the DE. The actual time is probably longer, since the repeated pulsed excitation shortens the polarization decay time.

\section{F. Control of the DE spin state using a picosecond optical pulse}

After demonstrating deterministic photogeneration of the $\mathrm{DE}$ in a well-defined pure state, here we present an experimental demonstration of coherent control ("rotation") of the DE state using a short optical pulse. We rotate the DE state from the eignestate in which it was photogenerated to a coherent superposition of its two eigenstates using a $\sim 10$ psec pulse, which is more than 5 orders of magnitude shorter than the measured lifetime of the DE and at least 4 orders of magnitude shorter than the DE coherence time.

Figure 6 presents schematically [Fig. 6(a)] the rotation of the DE spin state on the Bloch sphere during this experiment and [Fig. 6(b)] the sequence of pulses used to experimentally demonstrate the rotation. The pump pulse deterministically writes the DE in its lower-energy eigenstate, represented by the north pole of the Bloch sphere in Fig. 6(a). An eigenstate does not evolve in time, and the DE remains in this eigenstate as it radiatively decays. For use as a qubit, it must be possible to control the spin state of the $\mathrm{DE}$ and to rotate this state at will to any point on the Bloch sphere. We do this similarly to the way by which we $[13,14]$ and recently Muller et al. [15] controlled the BE spin state. A detuned $2 \pi$-area optical pulse is used to transfer the DE population through the $X X_{T \pm 3}^{0}$ resonance and back into the DE. During this transfer, the DE state acquires a relative phase difference between its two eigenstates that is dependent on the detuning from resonance of the optical pulse [34]. This phase accumulation is described on the Bloch
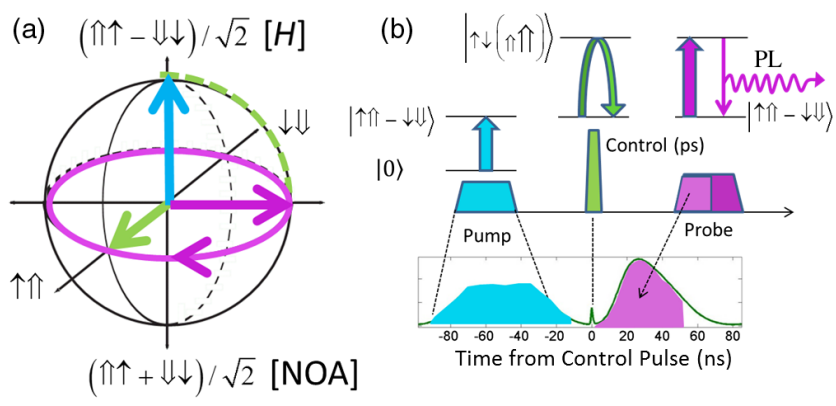

FIG. 6. (a) Schematic description of the precession of the DE on the Bloch sphere. The pump $\pi$ pulse [blue arrow, Fig. 3(b)] deterministically generates the DE in its lower-energy eigenstate (north pole of the sphere). The positively detuned circularly polarized control $2 \pi$ pulse [green arrow, Fig. 3(c)] "rotates" the $\mathrm{DE}$ by an angle of $\pi / 2$ about the polarization direction (green arrow) and brings the DE within the pulse duration (few picoseconds) to the equatorial plane of the Bloch sphere (dashed green line). The DE then precesses clockwise in time as described by the magenta trajectory on the Bloch sphere. The optical activity of the DE eigenstates ( $[H]$ for $H$ polarized and [NOA] for no optical activity) is shown to the right of the spin configuration. (b) Schematic description of the pulse sequence used in this experiment. A resonant DE $\pi$ pulse [blue arrow, Fig. 3(b)] is used to deterministically excite the DE in the $[H]$ eigenstate. Then, a properly detuned from the biexciton resonance, circularly polarized, $2 \pi$, picosecond pulse is used to rotate the DE to its $|+2\rangle$ coherent spin state. A long polarized probe pulse (magenta) is then used to probe the DE precession, which gives rise to temporal oscillations in the circular polarization of the emitted light from the biexciton transition [Fig. 3(d)]. The actual measured temporal sequence of pulses is shown below the schematic description.

sphere [Fig. 6(a)] as a rotation around the direction of the pulse circular polarization $[14,15]$. No detuning results in a $\pi$ rotation, while negative (positive) detuning results in larger (smaller) rotation angles $[14,15,34]$. Similarly to the separate carriers [7-10], full DE control in this manner requires two optical pulses.

Figure 6(b) shows the pulse sequence used in this experiment. After deterministic generation of the DE using a 60 -nsec $H$-polarized pulse, a very short ( $10 \mathrm{psec}) 2 \pi$ area $\sigma^{+}$-polarized control pulse, positively (negatively) detuned from the $X X_{T \pm 3}^{0}$ resonance, is applied to rotate the DE state $\pi / 2(3 \pi / 2)$ rad around the right-hand circularpolarization direction on the Bloch sphere. A $60 \mathrm{nsec}$ righthand circularly polarized $\mathrm{cw}$ probe is then used to reexcite the DE to the $X X_{T \pm 3}^{0}$ biexciton for measuring the time evolution of the DE spin state during these subsequent $60 \mathrm{nsec}$. The resulting $X X_{T \pm 3}^{0}$ emission is detected after a circular polarizer that matches the circular polarization of the probe pulse. By subtracting and dividing by the sum of the time-dependent signals from the cross and cocircular polarizations, the degree of circular polarization during the probe pulse is obtained. The entire sequence of pulses lasts $\sim 120 \mathrm{nsec}$ and the repetition rate is $1 \mathrm{MHz}$. In Fig. 6(b), the 


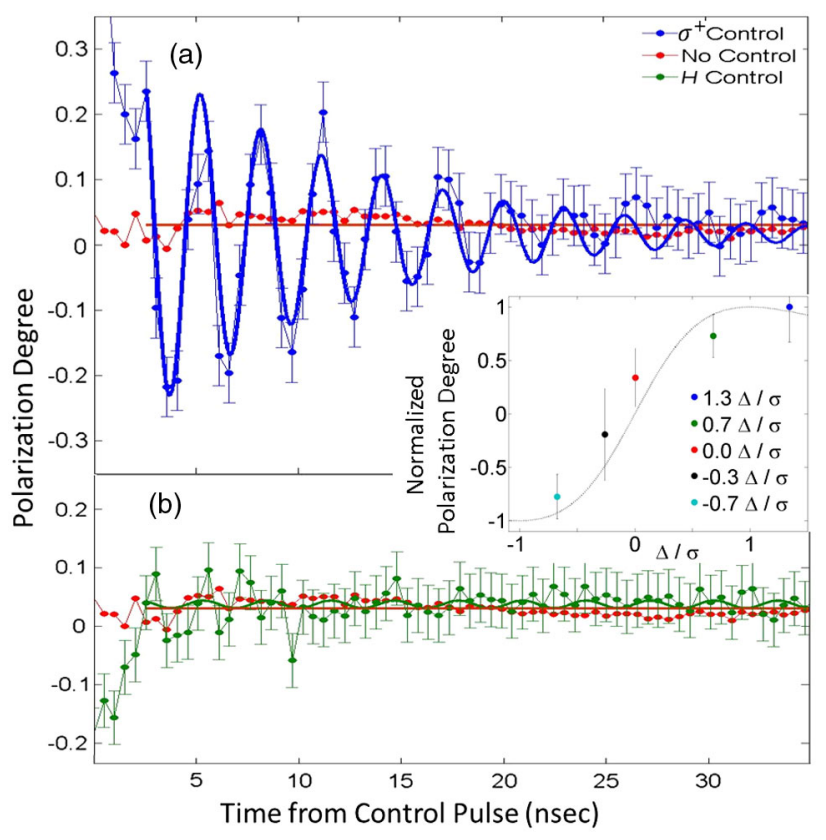

FIG. 7. Experimental demonstration of the DE spin control (a) [(b)] The measured (points) degree of circular polarization of the $X X_{T \pm 3}^{0}$ emission as a function of time after the application of a $\sigma^{+}$-polarized (right-hand circularly) $[H$-polarized (horizontal linearly)] control pulse (blue) [(green)], compared to that measured in the absence of a control pulse (red). The solid line in (a) represents a decaying sinusoidal model fit. Note that while no polarization oscillations are observed for the linearly polarized control pulse, they are clearly visible for the circularly polarized one. Points (line) in the inset show the measured (model) dependence of the normalized oscillation amplitude on the detuning.

measured pulse sequence is shown below the schematic description.

In Fig. 7(a), we present the measured (points) degree of circular polarization as a function of time after the control pulse during the first $35 \mathrm{nsec}$ of the probe pulse for a detuning energy of $\Delta / \sigma \approx 0.7$ for a $\sigma^{+}$-polarized control pulse. Here, $\Delta$ is the detuning from resonance and $\sigma=$ $100 \mu \mathrm{eV}$ is the full spectral width of the control laser at half maximum. The overlaid solid line presents a best-fit model of an exponentially decaying sinusoidal function $f(t)=P_{0} \sin \left(2 \pi t / \tau_{\mathrm{DE}}^{L}\right) \exp \left[-t / T_{\mathrm{PD}}\right]$, where $P_{0}$ is the initial polarization degree, $\tau_{\mathrm{DE}}^{L} \sim 3 \mathrm{nsec}$ is the DE precession time (Table I), and $T_{\mathrm{PD}}$ is a characteristic polarization decay time, which under these conditions of strong cw reexcitation is relatively short [only $\sim 20$ as opposed to $\sim 100 \mathrm{nsec}$ under pulsed excitation as in Fig. 5(d)].

In the inset of Fig. 7, points present the actual values of the best-fitted $P_{0}$ (normalized to 1 at maximum) as a function of the detuning energy. The solid line presents the theoretically expected dependence $P_{0}(\Delta)=$ $\sin (\pi-2 \arctan [\Delta / \sigma])$ [34]. The maximal degree of initial polarization achieved in these experiments at a detuning of $\Delta / \sigma \approx 1.0$ is about $30 \%$. Two factors limit the measured initial polarization in these experiments. The first is the temporal resolution of our detectors, which limits $\left|P_{0}\right|<0.6$. as discussed in Ref. [18]. The second factor is the repetition rate at which the experiments are conducted. The pulse-to-pulse separation is comparable to the DE lifetime. Therefore, in $\sim 40 \%$ of the cases, the residual DE population prevents the absorption of the pump pulse, thus further reducing $\left|P_{0}\right|$. A pulse sequence in which the QD is emptied after the probe pulse and before the DE is photogenerated again can, in principle, yield closer to unity fidelity.

We note in Fig. 7(a) that for a positive (negative, see inset) detuning there is a delay of $1 / 4(3 / 4)$ of a precession period between the control pulse time and the time at which the maximal polarization degree is obtained. This is an unambiguous experimental verification for the clockwise precession of the DE qubit [Fig. 6(a)]. This establishes experimentally that the optically active $\mathrm{DE}$ eigenstate, into which we deterministically initiate the DE, is the DE lowest energy eigenstate.

For completion, in Fig. 7(b) we present the measured degree of circular polarization at the same detuning as in Fig. 7(a), but this time the control pulse is linearly $H$ polarized. Clearly, as expected, no polarization oscillations are observed in this case, similar to the case with no control pulse at all (red line).

\section{CONCLUSIONS}

In conclusion, we demonstrate that the long-lived, quantum dot-confined dark exciton can be deterministically generated in a pure and well-defined spin eigenstate using a single optical pulse. We also show that its spin state can be coherently controlled using single picosecond pulse, resonantly tuned to or slightly detuned from a biexciton state. In addition, we obtain lower bounds of about 1 and $0.1 \mu \mathrm{sec}$ on the lifetime and coherence time of the dark exciton, respectively.

These demonstrations suggest that the dark exciton may have possible advantages over its constituents, the separate charge carrier as QD confined spin qubit. (a) The DE qubit requires no external magnetic field. However, a vertically applied magnetic field can be easily applied to the DE qubit, thereby increasing or tuning its Larmor frequency. (b) The DE coherence time as measured without spin echo is at least an order of magnitude longer than that of its constituents, under similar conditions. (c) The DE can be initialized deterministically by one single optical pulse, unlike the separate carriers, which require optical pumping by sequences of a few pulses. (d) The DE can be reset (i.e., the QD be emptied from both carriers and prepared for new initialization) by one single voltage pulse [19], which can be applied to all the QDs in the sample. Resetting the separate carrier qubits requires optical pumping, which may differ from one QD to another, and, in principle, requires more time. (e) The optical control of the DE is 
performed through a biexcitonic state. This state has two nondegenerate optical transitions, facilitating resonant detection and resonant excitation at two different energies and preventing blinding the detectors by the exciting laser field. This is in clear contrast with the separate carriers, which require one resonant excitation into a charged exciton (trion) state.

There are clear similarities between the DE qubit and the separate carrier qubits; thus, operations that were demonstrated or proposed with charge qubits are likewise possible with the DE qubit. These include, in particular, entangling the DE spin with that of the emitted biexciton photon [35-38] and coupling one QD-confined DE qubit with another DE qubit, either by spin-spin interactions [39,40] or by microcavity photons [41-43].

These features and increased flexibility in the initialization, possible reset and control of the dark exciton spin qubit, make it a semiconductor-based solid-state spin qubit with great potential for basic scientific studies and novel applications.

\section{ACKNOWLEDGMENTS}

The support of the Israel Science Foundation (ISF), the Israel Ministry of Science and Technology (MOST), and the Technion's RBNI are gratefully acknowledged. I. S. and E. R. S. contributed equally to this work.

[1] D. P. DiVincenzo, The Physical Implementation of Quantum Computation, Fortschr. Phys. 48, 771 (2000).

[2] H. J. Kimble, The Quantum Internet, Nature (London) 453, 1023 (2008).

[3] K. Hennessy, A. Badolato, M. Winger, D. Gerace, M. Atature, S. Gulde, S. Falt, E. L. Hu, and A. Imamoglu, Quantum Nature of a Strongly Coupled Single Quantum Dot-Cavity System, Nature (London) 445, 896 (2007).

[4] J. L. O'Brien, A. Furusawa, and J. Vuckovic, Photonic Quantum Technologies, Nat. Photonics 3, 687 (2009).

[5] E. D. Kim, K. Truex, X. Xu, B. Sun, D. G. Steel, A. S. Bracker, D. Gammon, and L. J. Sham, Fast Spin Rotations by Optically Controlled Geometric Phases in a ChargeTunable InAs Quantum Dot, Phys. Rev. Lett. 104, 167401 (2010).

[6] D. Press, K. De Greve, P. L. McMahon, T. D. Ladd, B. Friess, C. Schneider, M. Kamp, S. Hofling, A. Forchel, and Y. Yamamoto, Ultrafast Optical Spin Echo in a Single Quantum Dot, Nat. Photonics 4, 367 (2010).

[7] A. J. Ramsay, S. J. Boyle, R. S. Kolodka, J. B. B. Oliveira, J. Skiba-Szymanska, H. Y. Liu, M. Hopkinson, A. M. Fox, and M.S. Skolnick, Fast Optical Preparation, Control, and Readout of a Single Quantum Dot Spin, Phys. Rev. Lett. 100, 197401 (2008).

[8] A. Greilich, S. G. Carter, D. Kim, A. S. Bracker, and D. Gammon, Optical Control of One and Two Hole Spins in Interacting Quantum Dots, Nat. Photonics 5, 702 (2011).
[9] K. DeGreve, P. L. McMahon, D. Press, T. D. Ladd, D. Bisping, C. Schneider, M. Kamp, L. Worschech, S. Hofling, A. Forchel, and Y. Yamamoto, Ultrafast Coherent Control and Suppressed Nuclear Feedback of a Single Quantum Dot Hole Qubit, Nat. Phys. 7, 872 (2011).

[10] T. M. Godden, J. H. Quilter, A. J. Ramsay, Y. Wu, P. Brereton, S. J. Boyle, I. J. Luxmoore, J. Puebla-Nunez, A. M. Fox, and M. S. Skolnick, Coherent Optical Control of the Spin of a Single Hole in an InAs/GaAs Quantum Dot, Phys. Rev. Lett. 108, 017402 (2012).

[11] Peter Yu and Manual Cardona, Fundamentals of Semiconductors: Physics and Materials Properties, 4th ed. (Springer, Berlin, Germany, 2010).

[12] Y. Benny, S. Khatsevich, Y. Kodriano, E. Poem, R. Presman, D. Galushko, P. M. Petroff, and D. Gershoni, Coherent Optical Writing and Reading of the Exciton Spin State in Single Quantum Dots, Phys. Rev. Lett. 106, 040504 (2011).

[13] E. Poem, O. Kenneth, Y. Kodriano, Y. Benny, S. Khatsevich, J. E. Avron, and D. Gershoni, Optically Induced Rotation of an Exciton Spin in a Semiconductor Quantum Dot, Phys. Rev. Lett. 107, 087401 (2011).

[14] Y. Kodriano, I. Schwartz, E. Poem, Y. Benny, R. Presman, T. A. Truong, P. M. Petroff, and D. Gershoni, Complete Control of a Matter Qubit Using a Single Picosecond Laser Pulse, Phys. Rev. B 85, 241304 (2012).

[15] K. Muller, T. Kaldewey, R. Ripszam, J. S. Wildmann, A. Bechtold, M. Bichler, G. Koblmuller, G. Abstreiter, and J. J. Finley, All Optical Quantum Control of a Spin-Quantum State and Ultrafast Transduction into an Electric Current, Sci. Rep. 3, 1096 (2013).

[16] J. Fischer, W. A. Coish, D. V. Bulaev, and D. Loss, Spin Decoherence of a Heavy Hole Coupled to Nuclear Spins in a Quantum Dot, Phys. Rev. B 78, 155329 (2008).

[17] B. D. Gerardot, D. Brunner, P. A. Dalgarno, P. Ohberg, S. Seidl, M. Kroner, K. Karrai, N. G. Stoltz, P. M. Petroff, and R. J. Warburton, Optical Pumping of a Single Hole Spin in a Quantum Dot, Nature (London) 451, 441 (2008).

[18] E. Poem, Y. Kodriano, C. Tradonsky, N. H. Lindner, B. D. Gerardot, P. M. Petroff, and D. Gershoni, Accessing the Dark Exciton with Light, Nat. Phys. 6, 993 (2010).

[19] J. McFarlane, P. A. Dalgarno, B. D. Gerardot, R. H. Hadfield, R. J. Warburton, K. Karrai, A. Badolato, and P. M. Petroff, Gigahertz Bandwidth Electrical Control over a Dark Exciton-Based Memory Bit in a Single Quantum Dot, Appl. Phys. Lett. 94, 093113 (2009).

[20] G. Ramon, U. Mizrahi, N. Akopian, S. Braitbart, D. Gershoni, T. L. Reinecke, B. Gerardot, and P. M. Petroff, Emission Characteristics of Quantum Dots in Planar Microcavities, Phys. Rev. B 73, 205330 (2006).

[21] Y. Benny, Y. Kodriano, E. Poem, S. Khatsevitch, D. Gershoni, and P. M. Petroff, Two-Photon Photoluminescence Excitation Spectroscopy of Single Quantum Dots, Phys. Rev. B 84, 075473 (2011).

[22] Y. Kodriano, E. Poem, N. H. Lindner, C. Tradonsky, B. D. Gerardot, P. M. Petroff, J.E. Avron, and D. Gershoni, Radiative Cascade from Quantum Dot Metastable SpinBlockaded Biexciton, Phys. Rev. B 82, 155329 (2010).

[23] S. Alon-Braitbart, E. Poem, L. Fradkin, N. Akopian, S. Vilan, E. Lifshitz, E. Ehrenfreund, D. Gershoni, B. D. Gerardot, A. Badolato, and P. M. Petroff, Magneto Optics 
of Single Photons Emitted from Single InAs/GaAs SelfAssembled Quantum Dots in a Planar Microcavity, Physica (Amsterdam) 32E, 127 (2006).

[24] M. Bayer et al., Fine Structure of Neutral and Charged Excitons in Self-Assembled $\mathrm{In}(\mathrm{Ga}) \mathrm{As} /(\mathrm{Al}) \mathrm{GaAs}$ Quantum Dots, Phys. Rev. B 65, 195315 (2002).

[25] G. Ortner, M. Bayer, A. Larionov, V. Timofeev, A. Forchel, Y. Lyanda-Geller, T. Reinecke, P. Hawrylak, S. Fafard, and Z. Wasilewski, Fine Structure of Excitons in InAs/GaAs Coupled Quantum Dots: A Sensitive Test of Electronic Coupling, Phys. Rev. Lett. 90, 086404 (2003).

[26] E. L. Ivchenko, Optical Spectroscopy of Semiconductor Nanostructures (Alpha Science, Oxford, UK, 2005).

[27] M. A. Dupertuis, K. F. Karlsson, D. Y. Oberli, E. Pelucchi, A. Rudra, P. O. Holtz, and E. Kapon, Symmetries and the Polarized Optical Spectra of Exciton Complexes in Quantum Dots, Phys. Rev. Lett. 107, 127403 (2011).

[28] T. Smoleński, T. Kazimierczuk, M. Goryca, T. Jakubczyk, Ł. Kłopotowski, Ł. Cywiński, P. Wojnar, A. Golnik, and P. Kossacki, In-Plane Radiative Recombination Channel of a Dark Exciton in Self-Assembled Quantum Dots, Phys. Rev. B 86, 241305 (2012).

[29] M. Korkusinski and P. Hawrylak, Atomistic Theory of Emission from Dark Excitons in Self-Assembled Quantum Dots, Phys. Rev. B 87, 115310 (2013).

[30] M. Zielinski, Valence Band Offset, Strain and Shape Effects on Confined States in Self-Assembled InAs/InP and InAs/GaAs Quantum Dots, J. Phys. Condens. Matter 25, 465301 (2013).

[31] M. Zielinski, Y. Don, and D. Gershoni, Atomistic Theory of Dark Excitons in Self-Assembled Quantum Dots, arXiv:1411.5655.

[32] E. Poem, J. Shemesh, I. Marderfeld, D. Galushko, N. Akopian, D. Gershoni, B. D. Gerardot, A. Badolato, and P. M. Petroff, Polarization Sensitive Spectroscopy of Charged Quantum Dots, Phys. Rev. B 76, 235304 (2007).

[33] E. Poem, Y. Kodriano, C. Tradonsky, B. D. Gerardot, P. M. Petroff, and D. Gershoni, Radiative Cascades from Charged Semiconductor Quantum Dots, Phys. Rev. B 81, 085306 (2010).
[34] S. E. Economou and T. L. Reinecke, Theory of Fast Optical Spin Rotation in a Quantum Dot Based on Geometric Phases and Trapped States, Phys. Rev. Lett. 99, 217401 (2007).

[35] N. Akopian, N. H. Lindner, E. Poem, Y. Berlatzky, J. E. Avron, D. Gershoni, B. D. Gerardot, and P. M. Petroff, Entangled Photon Pairs from Semiconductor Quantum Dots, Phys. Rev. Lett. 96, 130501 (2006).

[36] W. B. Gao, P. Fallahi, E. Togan, J. Miguel-Sanchez, and A. Imamoglu, Observation of Entanglement between a Quantum Dot Spin and a Single Photon, Nature (London) 491, 426 (2012).

[37] K. DeGreve et al., Quantum-Dot Spin-Photon Entanglement via Frequency Downconversion to Telecom Wavelength, Nature (London) 491, 421 (2012).

[38] J. R. Schaibley, A. P. Burgers, G. A. McCracken, L.-M. Duan, P. R. Berman, D. G. Steel, A. S. Bracker, D. Gammon, and L. J. Sham, Demonstration of Quantum Entanglement between a Single Electron Spin Confined to an InAs Quantum Dot and a Photon, Phys. Rev. Lett. 110, 167401 (2013).

[39] M. Bayer, P. Hawrylak, K. Hinzer, S. Fafard, M. Korkusinski, Z. R. Wasilewski, O. Stern, and A. Forchel, Coupling and Entangling of Quantum States in Quantum Dot Molecules, Science 291, 451 (2001).

[40] O. Gywat, G. Burkard, and D. Loss, Biexcitons in Coupled Quantum Dots as a Source of Entangled Photons, Phys. Rev. B 65, 205329 (2002).

[41] A. Imamoglu, D. D. Awschalom, G. Burkard, D. P. DiVincenzo, D. Loss, M. Sherwin, and A. Small, Quantum Information Processing Using Quantum Dot Spins and Cavity QED, Phys. Rev. Lett. 83, 4204 (1999).

[42] D. Press, S. Götzinger, S. Reitzenstein, C. Hofmann, A. Löffler, M. Kamp, A. Forchel, and Y. Yamamoto, Photon Antibunching from a Single Quantum-Dot-Microcavity System in the Strong Coupling Regime, Phys. Rev. Lett. 98, 117402 (2007).

[43] C. Y. Hu, W. J. Munro, and J. G. Rarity, Deterministic Photon Entangler Using a Charged Quantum Dot inside a Microcavity, Phys. Rev. B 78, 125318 (2008). 